\title{
Análise das práticas de gestão do conhecimento de base tecnológica e funcional em uma clínica de imagem
}

\author{
Lidiana Antonioli Dal Bem Pires Mestre em Gestão do Conhecimento. Centro Universitário de Maringá (UNICESUMAR) - \\ Brasil. lidianaantonioli@yahoo.com.br \\ Arthur Gualberto Bacelar da Cruz Urpia Doutor em Economia. Universidade Federal do Rio de Janeiro (UFRI). \\ arthurbacellar@yahoo.com.br \\ Ely Mitie Massuda Doutora em História. Centro Universitário de Maringá (UNICESUMAR) - Brasil. \\ ely.massuda@unicesumar.edu.br
}

\section{RESUMO}

Com os avanços nas tecnologias das informações e comunicações (TICS) na década de 1990, dá-se início ao que se denomina 'Era da informação e do conhecimento', em que a informação e o conhecimento passam a se constituir recursos vitais para as organizações. $\mathrm{Na}$ área de saúde, intensas transformações veem ocorrendo, transpondo os domínios que lhe são intrínsecos, mudando, assim, os paradigmas de tomadas de decisão pelos gestores. Devido a sua complexidade e o envolvimento das múltiplas áreas de conhecimento e atores que envolvem a área da área da saúde, a Gestão do Conhecimento (GC) adquire caráter estratégico, trazendo-Ihe importantes contribuições. Diante disso, o objetivo dessa pesquisa foi diagnosticar o nível de implantação e de alcance das práticas de GC de Base Tecnológica e Funcional em uma empresa do ramo de saúde que realiza exames de diagnóstico por imagem na cidade de Maringá (PR). A pesquisa de caráter descritivo, de abordagem quantitativa e qualitativa, foi realizada por meio de estudo de caso e análise documental de registros da empresa. Para a coleta de dados foi aplicado um questionário desenvolvido pelo Instituto de Pesquisa Econômica Aplicada (IPEA). Como resultado, observou-se que o nível efetivo de implantação e o nível de amplitude do alcance das práticas de GC de Base Tecnológica e Funcional foram altos e que apenas a prática Enterprise Resource Planning está sendo utilizada abaixo de suas potencialidades. Diante desse contexto, algumas medidas e ações foram sugeridas a fim de consolidar ainda mais as práticas de GC de base tecnológica e funcional.

Palavras-chave: Saúde. Gestão do Conhecimento. Práticas de Gestão do Conhecimento.

\section{Analysis of the practices of knowledge management of technological and functional basis in image clinic}

\begin{abstract}
With advances in information and communication technologies (ICTs) in the 1990s, the so-called 'information and knowledge age' begins, in which information and knowledge become vital resources for organizations. In the area of health, intense transformations are occurring, transposing the domains that are intrinsic to it, thus changing the paradigms of decision making by managers. Due to its complexity and the involvement of the multiple areas of knowledge and actors that involve the area of health, Knowledge Management (KM) acquires strategic character, bringing important contributions. Therefore, the objective of this research was to diagnose the level of implantation and outreach of the Technological and Functional Base GC practices in a health care company that performs diagnostic imaging tests in the city of Maringá (PR). The descriptive, quantitative and qualitative research was carried out through a case study and documentary analysis of company records. For data collection, a questionnaire was developed by the Institute of Applied Economic Research (IPEA). As a result, it was observed that the effective level of implementation and the breadth of reach of KM practices on a Technological and Functional basis were high and that only the Enterprise Resource Planning practice is being used below its potential. Given this context, some measures and actions have been suggested in order to further consolidate the technological and functional KM practices.
\end{abstract}

Keywords: Health. Knowledge management. Practices of Knowledge Management. 


\section{INTRODUÇÃO}

Várias mudanças econômicas, tecnológicas e sociais vêm ocorrendo nas últimas décadas, algo que provocou uma mudança no padrão de acumulação do capital. A partir da década de 1990, com os avanços nas tecnologias das informações e comunicações (TICS), dá-se início à 'Era da informação e do conhecimento'. Para tal foi de extrema importância os avanços na área da microeletrônica com o chip microeletrônico, softwarese instrumentos de controle. Na 'Era da informação e do conhecimento' a importância dos investimentos em inovação tecnológica está relacionada ao fato de que o conhecimento é o meio essencial para a competitividade na nova economia mundial.

Dessa forma, é necessário que as organizações gerem o conhecimento, pois este é um dos principais ativos para acumulação do capital. Para geri-lo é fundamental aplicar a Gestão do Conhecimento (GC). A GC surgiu como um conjunto de métodos, conceitos e atividades para agregar valor ao capital intelectual e, com isso, atingir sua capacidade de criar, armazenar, recuperar, transferir e reutilizar o conhecimento organizacional (DALKIR, 2011).

Em relação à área de saúde, verifica-se que os paradigmas da prestação de serviços nessa área estão sendo intensamente questionados, tendo-se em vista que ambientes complexos e dinâmicos envolvem áreas de conhecimento e múltiplos atores, além de possibilidades para tomar decisão para os principais problemas como: a qualidade dos serviços prestados; carência de profissionais, principalmente médicos, nas Unidades Básicas de Saúde (UBS) e em hospitais; problemas nas Unidades de Pronto Atendimento (UPA); atrasos na liberação de exames e distribuição de medicamentos gratuitos. No âmbito gerencial também há a inquietação por parte de gestores em como ampliar seu capital intelectual (ROSENBERG; OHAYON; BATISTA, 2008).

Diante da complexidade que envolve a área da saúde, observa-se que nas organizações do ramo, a GC adquire caráter estratégico, trazendo-lhes importantes contribuições, tais como: melhora o convívio entre os colaboradores; amplia a capacidade das organizações obterem informações; o conhecimento passa a ser compartilhado entre os usuários de forma mais eficiente e melhora o seu armazenamento (ROSENBERG; OHAYON; BATISTA, 2008).

Algumas empresas da área como a Unimed, que é uma operadora de planos de saúde, vêm adotando determinadas práticas de GC, como Gestão por Competências e Portais, além de oferecer um curso de GC com a finalidade de instruir dirigentes, cooperados e colaboradores em geral por meio da Faculdade Unimed. No setor público, algumas práticas têm sido empregadas a exemplo da Secretaria de Saúde de Maringá (PR), que possui também um portal onde as informações estão disponíveis para todos os usuários, além de outras. No entanto, pouco tem se investigado sobre as práticas de GC alocadas na área de saúde, principalmente em clínicas laboratoriais que realizam exames por imagem.

Diante disso, o objetivo geral deste trabalho é diagnosticar o nível de implantação e de alcance de práticas de GC de base tecnológica e funcional em uma empresa do ramo de saúde que realiza exames de diagnóstico por imagem na cidade de Maringá. As práticas de GC de base tecnológica dão suporte à GC organizacional, incluindo a automação da gestão da informação, dos aplicativos e das ferramentas de Tecnologia da Informação (TI) para captura, difusão e colaboração (BATISTA, 2006). Ou seja, as práticas de GC de base tecnológica, apesar de não refletirem o nível de maturidade em GC da empresa como um todo, são de grande importância para a aplicação da GC no âmbito organizacional, o que justifica a necessidade de uma análise sobre estas práticas.

O presente estudo também se justifica por permitir conhecer o nível das práticas de GC de base tecnológica da empresa que realiza exames de imagem do município de Maringá, para mais adiante identificar práticas que possam ser aplicadas com o intuito de consolidar processos de GC nessas instituições. "Identificar as práticas mais frequentes e as menos comuns é relevante para o planejamento de ações de sensibilização e capacitação sobre GC nas organizações analisadas" (BATISTA, 2006, p. 9). Por outro lado, a natureza dos serviços disponibilizados pela empresa ressalta seu papel no bem-estar da população. Por meio destes serviços torna-se possível a realização de diagnósticos da enfermidade do indivíduo pelos profissionais da área da saúde. 
Este artigo, além desta breve introdução, contém mais quatro seções. A segunda seção apresenta uma contextualização sobre a gestão do conhecimento e sobre as práticas da base tecnológica e funcional. A terceira seção traz todo o procedimento metodológico da pesquisa. A quarta seção realiza a apresentação dos dados e análise dos resultados. Por fim, a quinta seção aponta as consideraçõ es finais.

\section{A GESTÃO DO CONHECIMENTO: CONCEITOS E PRÁTICAS DE BASE TECNOLÓGICA E FUNCIONAL}

Este capítulo traz o conceito de Gestão do Conhecimento (GC) e aborda as práticas de base tecnológica e funcional.

\subsection{A Gestão do Conhecimento - GC}

Em meados de 1960, Drucker (1981) já mencionava que o conhecimento era a base para determinados setores como os de produção, informação e serviços, sendo de responsabilidade das empresas criá-lo de diversas formas. Logo, os termos adotados por Drucker, tais como: "trabalhador do conhecimento" e "sociedade do conhecimento", deixam claro a importância do conhecimento para empresas da sociedade pós-capitalista. Entretanto, o termo Gestão do Conhecimento (Knowledge Management), segundo Souza (2014), foi expresso pela primeira vez nos Estados Unidos por Karl Wiig, em 1986. Em seguida, destaca-se o trabalho de Takeuchi e Nonaka, em 1987, no Japão, que objetivou compreender as pesquisas de Polanyi e aplicá-las nas organizações.

Dalkir (2011) definiu GC como a incorporação tanto do armazenamento quanto da captura do conhecimento atrelado à valorização de ativos intelectuais. Rodriguez (2011) explica que a GC pode ser conceituada como a habilidade que uma organização possui em criar o conhecimento e articular sua disseminação interna e absorvê-la em seus sistemas, produtos e serviços. Davenport e Prusak (1999) informam que a GC deve-se iniciar por um problema que já existe na organização e que está relacionado ao conhecimento. Produtos mal elaborados, redução de contratos negociados e perdas de clientes são exemplos de má utilização da GC pelas empresas.

Na visão de Dalkir (2011), a GC pode fornecer benefícios às comunidades de práticas, aos colaboradores e para as organizações. Para as pessoas pode proporcionar os seguintes benefícios: realização de tarefas sem desperdícios de tempo por meio de melhores soluções de problemas e tomada de decisão; os indivíduos se mantêm atualizados; e ainda, as pessoas obtêm laços comunitários dentro da empresa. Para as comunidades de prática, têm-se os seguintes benefícios: fortalecimento de uma linguagem comum; ampliação de código de ética profissional onde todos possam seguir. A utilização e a ascensão da GC nas organizações são apontadas como fatores necessários na procura por competitividade, pois se torna responsável por pequenas modificações, tais como: informatização de uma empresa ou aperfeiçoamento nos postos de trabalho; repartição de tarefas adequadas no setor produtivo, abrangendo transformações no ambiente de produção; resolução de problemas agilmente; maior agregação do conhecimento em serviços e produtos; expansão das melhores práticas; preparação das empresas para a concorrência e ampliação das oportunidades para inovação.

Por meio da GC, a empresa consegue monitorar e se apossar dos conhecimentos dos indivíduos para alcançar as metas da organização e prover os processos, isto mediante o uso de portais do conhecimento, mapeamento de competências, mapeamento de processos, dentre outros.

Logo, a GC visa suprir as necessidades organizacionais, uma vez que a organização é composta de pessoas dotadas de conhecimento e elas precisam gerá-lo, disseminá-lo e compartilhá-lo para gerir recursos e manter-se competitiva no mercado. No entanto, vale salientar que a expansão da vantagem competitiva de uma organização é diretamente proporcional às limitações que outras têm para imitar seu conhecimento (TARAPANOFF, 2006).

O processo de GC, em si, é uma atividade independente, mas, quando ligada ao processo decisório, está fortemente ligado ao processo de gestão da informação e ao trabalho e análise da informação. A inteligência (estratégica) pode ser considerada síntese do processo de 
trabalho da informação e do conhecimento, gerando conhecimento novo capaz de indicar novos caminhos para a empresa, a inovação em si éinteligência também (TARAPANOFF, 2006, p. 30)

Dentro de uma organização para que ocorra planejamento estratégico e tomada de decisão é necessário que os componentes inteligência, informação e conhecimento estejam inseridos nos processos de gestão (TARAPANOFF, 2006). O planejamento estratégico tem por intuito amplificar e conservar uma adaptação entre os recursos da empresa e os objetivos, além das mudanças de oportunidades de negócio. Através das estratégias, as organizações recorrem aos seus pontos fortes a fim de usufruírem das oportunidades e efetivar ações que reduzam o choque das ameaças sob seus pontos fracos. A inteligência também é necessária, tanto na fase inicial da estratégia quanto na fase de execução e integração.

Souza (2014) afirma que, no Brasil, os precursores da GC foram J osé Cláudio Cyrineu e J ayme Teixeira Filho. A GC teve seu início com o objetivo de agregar valor à informação e melhorar o seu fluxo participativo em toda empresa, a fim de garantir a competitividade no mercado.

A empresa Petrobrás decidiu estruturar a área de GC no ano de 2000 e em conjunto com outras empresas, tanto estrangeiras como nacionais, participaram de sessões de workshops com esse propósito resultando em um primeiro modelo de GC para a empresa. Dessa forma, a Petrobrás instituiu formalmente a gerência corporativa de GC, o que levou outras empresas a tomarem iniciativas semelhantes (RODRIGUEZ, 2011).

Entre os períodos de 2000 a 2007, algumas empresas formaram gerências direcionadas ao conhecimento e inovação, a exemplo do Banco Nacional de Desenvolvimento (BNDES), Gerdau, Correios, Vale do Rio Doce e o Tribunal de Justiça. A partir desses eventos, surge no Brasil a Sociedade Brasileira de GC (SBGC) com o intuito de agregar os esforços por meio de seminários, palestras e grupo de debates. Na área acadêmica surge um centro de pesquisa multidisciplinar em GC na Universidade Federal de Santa Catarina (UFSC) (RODRIGUEZ, 2011).

\subsection{Práticas de GC de base tecnológica e funcional}

Na literatura, o uso dos termos práticas ${ }^{1}$ e ferramentas de GC é imprecisa. Alguns autores, como Steil (2007) e Batista (2006) privilegiam o termo práticas de GC enquanto Dalkir (2005; 2011), Uriarte Jr (2008) e Young (2010), utilizam o termo ferramentas de GC. Para a presente pesquisa, será utilizado o primeiro termo, pois para o estudo de caso serão aplicadas as práticas de GC apresentadas no IPEA.

As práticas de GC de base tecnológica e funcional estão ligadas ao eixo tecnológico e funcional, sendo utilizadas como suporte para a GC organizacional, compondo a automação dos aplicativos e das ferramentas de tecnologia da informação, com foco na captura, difusão e cooperação (BATISTA, 2006).

Portais/ intranets/ extranets nada mais são do que uma forma de armazenamento e disseminação do conhecimento empresarial nos processos de negócios, procedimentos, políticas e outros tipos de conhecimentos que foram codificados, conforme mencionado por Dalkir (2011). As Extranets contribuem que fornecedores, clientes, consultores, entre outros, acessem a sites de rede direcionados a banco de dados de outras organizações a fim de obterem mais informações. Essa prática tem como vantagens e aplicações a agilidade em transmitir dados e, dessa forma, descentraliza atividades, desloca competências e organiza a combinação do conhecimento (DUARTE et al., 2006).

Logo, os portais, intranets e extranets são um conjunto de práticas localizados na web com repertório de conhecimento direcionado aos funcionários e toda a organização, com o intuito de possibilitar úteis informações. Assim, eles servem para oferecer a criação do conhecimento a partir de um espaço virtual.

Sistemas de Workflow são considerados como prática voltada ao controle de qualidade de informação sustentada pelo mecanismo do fluxo ou canal de documentos. Essa prática tecnológica garante a

\footnotetext{
${ }^{1}$ A palavra 'prática' é conceituada no dicionário online Infopédia (2016) como: 1. Atividade que visa a obtenção de resultados concretos. 2. Aplicação das regras e dos princípios de uma arte ou ciência; 3. Maneira concreta de exercer uma arte ou conhecimento; experiência; exercício.
} 
captação da "inteligência" de um determinado procedimento, por meio de sua geração, automatização e controle (DUARTE et al., 2006). Vale salientar que os Sistemas de Workflow são delineados para automatizar os processos e devem ser colocados como um ativo diferenciado na conversão do conhecimento tácito em explícito, além disso, é um tipo de sistema de informação considerando de forma mais abrangente.

Gestão Eletrônica de Documentos (GED) é outra prática utilizada na tecnologia da informação que, através de aplicativos, controlam a edição, emissão, além de acompanhar a tramitação, divisão, armazenamento e descarte de documentos. Ainda pode-se afirmar que a GED é uma caracterização de software e equipamentos, geralmente de recursos de telecomunicação alicerçado em computador e automatizada, que arquiva e capta imagens, portanto, pode ser lida por máquinas e acionadas por computador para sua regeneração (AVEDON, 2002).

O GED pode converter informações, podendo ser caracterizado por imagens, textos e voz para um formato digital. Essa prática é recomendada para conduzir o ciclo de vida das informações desde o momento da criação até o seu armazenamento. Roese e Ladeira Júnior (2009) alegam que a prática GED vem sendo utilizada desde 1990 por grandes empresas petrolíferas, tais como a Petrolífera Britânica e a Shell.

Customer Relationship Management (CRM) é uma prática de GC voltada para a relação entre as organizações e seus clientes. Para Carvalho (2012, p. 49):

Sistema de CRM: possibilita a criação de uma estrutura que integra e automatiza diversos processos de atendimento ao cliente, marketing e serviços de produtos relacionados aos clientes de uma organização com o intuito de ajudá-la a identificar e escolher seus melhores clientes para desenvolver uma relação forte e duradoura com eles.

Enterprise Resource Planning (ERP), também conhecido como sistemas integrados de gestão, reúne todas as informações contidas na organização por meio de uma única base de dados. Para Gambôa et al. (2004), o ERP nada mais é do que um pacote comercial de software capaz de organizar, uniformizar e incluir as informações que rodeiam pelas empresas. Ainda para os autores, os ERPs surgiram na década de 90 para auxiliar as organizações a melhorar a produtividade e, consequentemente, obter vantagens competitivas por meio da utilização da Tecnologia da Informação (TI).

Para Mendes e Escrivão Filho (2002), quando uma empresa adota um ERP, o objetivo fundamental não é simplesmente inserir um software em produção, mas ap rimorar os processos de negócios fazendo o uso da tecnologia da informação. Na verdade, ao implementar o ERP, verifica-se na empresa um processo de mudança organizacional e não simplesmente uma mudança tecnológica.

\section{METODOLOGIA}

Essa seção visa apresentar os procedimentos metodológicos que foram aplicados na efetivação da pesquisa. Para dar sustentação ao desenvolvimento desse artigo, fez-se necessário, inicialmente, realizar levantamentos bibliográficos e também foi realizada uma análise documental de registros da empresa. Esta pesquisa pode ser classificada como de caráter descritivo, possuindo abordagens quantitativa e qualitativa, e adota o procedimento técnico de estudo de caso.

Foram analisadas sete unidades de clínicas de imagem e o escritório administrativo que pertencem à mesma empresa, localizada em Maringá, na região norte do Paraná. Todas as unidades situam-se no mesmo município. Todas as sete unidades da empresa realizam exames laboratoriais e de imagem, quais sejam: ultrassom, raio $x$, tomografia e ressonância. Para este estudo, foram selecionad os os setores que correspondem aos serviços de imagem. Responderam ao instrumento um total de 14 pessoas e a pesquisa abrangeu toda a população de gestores da empresa de Diagnóstico por Imagem. Faz-se importante salientar que os respondentes informaram não possuir experiência e contato com conceitos de GC, até por isso os pesquisadores fizeram esclarecimentos sobre as práticas de GC no momento da coleta dos dados. 0 Quadro 1 apresenta o perfil dos respondentes ao destacar os seus cargos e atividades exercidas. 
Quadro 1 - Perfil dos respondentes

\begin{tabular}{|l|l|}
\hline \multicolumn{1}{|c|}{ Cargo } & \multicolumn{1}{c|}{ Atividades exercidas } \\
\hline Gestor Recursos Humanos & $\begin{array}{l}\text { Responsável por supervisionar os colaboradores do setor, além de } \\
\text { exercer as seguintes atividades: contratação, demissão, elaborar } \\
\text { políticas de cargo e salários, treinamentos. }\end{array}$ \\
\hline $\begin{array}{l}\text { Gestor de Agendamento } \\
\text { de exames }\end{array}$ & $\begin{array}{l}\text { Supervisiona todas as unidades da empresa de Maringá em relação ao } \\
\text { agendamento local e via telefone dos exames. }\end{array}$ \\
\hline Gestor Financeiro & $\begin{array}{l}\text { Responsável por efetuar os pagamentos de todas as unidades e contas } \\
\text { a receber apenas dos pacientes particulares. }\end{array}$ \\
\hline $\begin{array}{l}\text { Gestor Faturamento/ } \\
\text { Contas a receber }\end{array}$ & $\begin{array}{l}\text { Responsável por coordenar o setor de faturamento dos serviços } \\
\text { prestados aos hospitais, clínicas e pacientes além de receber os } \\
\text { pagamentos realizados pelos convênios. }\end{array}$ \\
\hline $\begin{array}{l}\text { Gestor de Compras e } \\
\text { Manutenção }\end{array}$ & $\begin{array}{l}\text { Responsável por efetuar as compras desde materiais de escritório, } \\
\text { limpeza a materiais e medicamentos (o gestor não realiza compras de } \\
\text { aparelhos de imagem e laboratoriais), além de ser responsável por fazer } \\
\text { manutenções gerais em todas as unidades. }\end{array}$ \\
\hline $\begin{array}{l}\text { Gestores do setor de } \\
\text { Recepção }\end{array}$ & $\begin{array}{l}\text { Responsáveis por supervisionar os colaboradores das áreas da da } \\
\text { recepção. }\end{array}$ \\
\hline $\begin{array}{l}\text { Gestor da área técnica de } \\
\text { Radiologia }\end{array}$ & $\begin{array}{l}\text { Responsável por efetuar compra de equipamentos de imagem e de } \\
\text { executar manutenções desses equipamentos. }\end{array}$ \\
\hline $\begin{array}{l}\text { Gestor da área de } \\
\text { Enfermagem }\end{array}$ & $\begin{array}{l}\text { Supervisiona os técnicos e auxiliares de enfermagem em recepcionare } \\
\text { posicionar o paciente na realização do exame. }\end{array}$ \\
\hline $\begin{array}{l}\text { Gestor de Tecnologia da } \\
\text { Informação }\end{array}$ & $\begin{array}{l}\text { Responsável pela manutenção e elaboração dos programas de } \\
\text { softwaresutilizados nas unidades. }\end{array}$ \\
\hline $\begin{array}{l}\text { Gestor de Administração } \\
\text { Geral }\end{array}$ & $\begin{array}{l}\text { Responsável por gerenciar todas as unidades, principalmente nos } \\
\text { assuntos pertinentes à administração. }\end{array}$ \\
\hline Diretor & Médico e proprietário da empresa. \\
\hline & \multicolumn{1}{|c|}{ Fonte: Elaborado pelos autores (2017). } \\
\hline
\end{tabular}

Para a coleta de dados, foi aplicado o questionário elaborado pelo Instituto de Pesquisa Econômica Aplicada (IPEA) ${ }^{2}$ que foi adaptado para fins da presente pesquisa. 0 questionário possibilita a realização do diagnóstico do grau de alcance e do estágio de implantação das práticas de GC na empresa. No presente trabalho foram analisadas as práticas pertencentes à categoria base tecnológica e funcional descritas no Quadro 2.

Quadro 2 - Classificação das práticas de GC utilizadas no questionário aplicado à empresa Clínica de Diagnóstico por Imagem.

\begin{tabular}{|c|l|}
\hline \multicolumn{1}{|c|}{ Dimensões das práticas de GC (adaptado) } & \multicolumn{1}{c|}{ Práticas } \\
\hline \multirow{4}{*}{ Práticas de GC de Recursos Humanos } & Comunidades de Prática \\
& Coaching \\
& Fóruns \\
& Educação Corporativa \\
& Narrativas \\
\hline & Benchmarking interno e externo \\
& Melhores Práticas \\
& Mapeamento do Conhecimento \\
& Sistema de Gestão por Competência \\
& Banco de Competências Individuais \\
& Memória Organizacional \\
& Sistema de Inteligência Organizacional \\
& Gestão do Capital Intelectual \\
\hline
\end{tabular}

20 questionário utilizado na pesquisa está disponível no site do IPEA (http://www.ipea.gov.br/portal/images/stories/PDFs/TDs/td_1316.pdf). 


\begin{tabular}{|c|c|}
\hline Práticas de GC de Base Tecnológica e Funcional & $\begin{array}{l}\text { Portais/intranets/extranets } \\
\text { Sistemas de Workflow } \\
\text { Gestão Eletrônica de Documentos (GED) } \\
\text { Costumer Relationship Management (CRM) } \\
\text { Enterprise Resource Planning (ERP) }\end{array}$ \\
\hline
\end{tabular}

Fonte: Elaborado pelos autores (2017).

Para averiguar o estágio de implantação das práticas de GC a seguinte escala foi utilizada: [0] Não existem planos para implementação da prática; [1] Existem ações planejadas para a implementação da prática no futuro; [2] A prática está em processo de implementação; [3] A prática já está implantada; [4] A prática já está implantada e apresenta resultados importantes e relevantes para a organização.

Para analisar o nível de alcance das práticas de GC considerou-se a seguinte escala: [1] Poucas iniciativas isoladas dentro da organização; [2] Alguns departamentos usam a prática; [3] Muitos departamentos usam a prática; [4] Amplamente disseminada na organização.

Vale ressaltar que para análise do resultado em relação ao nível efetivo de implantação das práticas e ferramentas selecionadas, foram analisadas apenas as respostas relacionadas aos níveis 3 (a prática já está implantada) e 4 (a prática já está implantada e apresenta resultados importantes e relevantes para a organização). Os demais níveis contribuíram para apurar a intenção da empresa em implementar as práticas futuramente (nível 1), se as práticas já estão em processo de implementação (nível 2), ou não há existência de planos para implantação das práticas (nível 0 ).

Em relação à análise dos resultados a respeito do nível de amplitude do alcance das práticas e ferramentas selecionadas, foram analisadas também apenas as respostas relacionadas aos níveis 3 (muitos departamentos usam a prática) e 4 (amplamente disseminada na organização) da escala do estágio do alcance, tendo-se em vista que apenas as respostas desses dois níveis indicam um amplo alcance das práticas e ferramentas. Os níveis 1 e 2 contribuíram para averiguar se há poucas iniciativas isoladas dentro da organização (nível 1) ou se a prática é utilizada apenas por poucos departamentos (nível 2).

Diante disso, para analisar o nível efetivo de implantação e nível de amplitude do alcance das práticas e ferramentas tal escala foi elaborada com os parâmetros sendo criados de forma arbitrária (Quadro 3).

Quadro 3 - Escala para análise dos resultados relativos ao nível efetivo de Implantação e estágio de amplitude do Alcance das práticas de GC.

\begin{tabular}{|c|c|}
\hline $\begin{array}{c}\text { Nível Efetivo de Implantação e de amplitude do } \\
\text { Alcance }\end{array}$ & $\mathbf{3 + 4}$ \\
\hline Nível baixo & Entre 0 a 39\% \\
\hline Nível médio & Entre $40 \%$ a $60 \%$ \\
\hline Nível alto & Acima de $60 \%$ \\
\hline
\end{tabular}

Fonte: Elaborado pelos autores (2017).

Para tabular os resultados do questionário, os dados foram organizados por meio do Programa Microsoft Excel, versão 2010, e apurados por meio de técnicas estatísticas. Com a finalidade de averiguar a confiabilidade dos dados obtidos pelo questionário calculou-se o coeficiente alfa de Cronbach. Segundo Hora, Monteiro e Arica (2010), esse método estatístico foi apresentado por Lee J. Cronbach em 1951 como um meio de valorizar a confiabilidade em um questionário aplicado em uma pesquisa. Ainda para Hora, Monteiro e Arica (2010), o alfa visa medir a correlação entre as respostas em um questionário por meio da análise do perfil das respostas relatadas pelos respondentes. Abaixo, pode-se verificar a equação para o cálculo do coeficiente alfa:

$$
\alpha=\left(\frac{k}{k-1}\right) \times\left(1-\frac{\sum_{i=1}^{k} s_{i}^{2}}{s_{t}^{2}}\right)
$$


Tem-se para os elementos da equação, a seguinte definição: $k$ : Número de itens/perguntas do questionário; $s_{i}^{2}$ : variância de cada item; e $s_{t}^{2}$ : total das variâncias dos respondentes. Para Matthiensen (2011), os valores apurados em a podem variar entre 0 e 1 . Quanto mais próximo de 1, a confiabilidade tornase maior entre os indicadores. Por outro lado, se os valores apurados em a forem próximo de 0 , entende-se que todos os itens de uma escala são independentes.

O Quadro 4 apresenta a escala adotada para análise da qualificação da consistência dos dados, conforme 0 alfa de Cronbach.

Quadro 4 - Qualificação da consistência, de acordo com o Alfa de Cronbach

\begin{tabular}{|c|c|}
\hline Intervalo & Consistência \\
\hline$a<0,6$ & Fraca \\
\hline $0,6 \leq a<0,7$ & Moderada \\
\hline $0,7 \leq a<0,8$ & Boa \\
\hline $0,8 \leq a<0,9$ & Muito Boa \\
\hline$a \geq 0,9$ & Excelente \\
\hline
\end{tabular}

Fonte: Elaborado pelos autores com base em Loesch e Hoeltgebaum (2012).

Conforme o Quadro 4, observa-se que para um alfa ser no mínimo satisfatório, ele deve apresentar um valor de 0,7. As determinadas regras podem ser seguidas para assegurar as credibilidades: i) para verificar um determinado conceito, a quantidade de itens de uma escala tem que ser igual ou superior a três; ii) caso 0 coeficiente alfa calculado for baixo, recomenda-se que sejam excluídos os itens de mensuração correlacionados com os outros a nível inferior a 0,3 . Quando se realiza o recálculo da confiabilidade, 0 valor de alfa poderá aumentar (LOESCH; HOELTGEBAUM, 2012). Os dados foram coletados entre os períodos de outubro de 2016 a fevereiro de 2017 através de visitas, aplicação de questionário, além da plataforma Whatsapp.

\section{APRESENTAÇÃO DOS DADOS E ANÁLISE DOS RESULTADOS}

Neste capítulo são apresentados os dados da pesquisa e realizadas as análises dos resultados.

\subsection{Alfa de Cronbach}

Como já foi indicado na Metodologia, fez-se necessário utilizar o coeficiente alfa de Cronbach a fim de medir a confiabilidade e consistência dos dados provenientes desta pesquisa. Para efetivação da análise de consistência foi realizada a tabulação dos dados de dezoito práticas contidas no instrumento de diagnóstico do IPEA e, em seguida, transformadas em itens de uma planilha. Contudo, para a presente pesquisa, selecionaram-se práticas que pertencem a categoria de base tecnológica e funcional.

O valor do alfa de Cronbach para os dezoito itens que representam o nível de implantação de todas as práticas contidas no instrumento de diagnóstico do IPEA foi de 0,8814 , sendo considerado um nível de consistência ótimo de acordo com a escala de Loesch e Hoeltgebaum (2012).

Já o valor do alfa de Cronbach para os dezoito itens que representam o nível de alcance foi de 0,8872 . De acordo com a escala de Loesch e Hoeltgebaum (2012), esse valor encontrado representa também um nível de consistência ótimo. 
Diante dos valores dos alfas de Cronbach encontrados e dado que o instrumento de diagnóstico utilizado foi validado em diversos trabalhos, concluí-se que a pesquisa apresenta uma grande robustez.

\subsection{Resultado da análise do diagnóstico e análise documental}

Nessa seção são apresentados os resultados obtidos com o diagnóstico referente às práticas de GC de base tecnológica e funcional, no que diz respeito aos níveis efetivos de implantação, níveis de amplitude do alcance e análise integrada desses níveis; além da análise documental.

Tabela 1 - Nível de Implantação das práticas de GC de base tecnológica e funcional

\begin{tabular}{|l|c|c|c|c|c|c|c|}
\hline \multirow{2}{*}{\multicolumn{1}{|c|}{ PRÁTICA }} & \multicolumn{3}{|c|}{} & & & & \\
\hline & & & & & \\
\hline & $\mathbf{0}$ & $\mathbf{1}$ & $\mathbf{2}$ & $\mathbf{3}$ & $\mathbf{4}$ & $\mathbf{N} / \mathbf{R}$ \\
\hline Ferramentas de colaboração & $0 \%$ & $0 \%$ & $7 \%$ & $50 \%$ & $43 \%$ & $0 \%$ \\
\hline Sistemas de workflow & $7 \%$ & $7 \%$ & $7 \%$ & $7 \%$ & $64 \%$ & $7 \%$ \\
\hline Gestão Eletrônica de Documentos (GED) & $0 \%$ & $0 \%$ & $36 \%$ & $14 \%$ & $50 \%$ & $0 \%$ \\
\hline Costumer Relationship Management (CRM) & $0 \%$ & $0 \%$ & $7 \%$ & $43 \%$ & $43 \%$ & $7 \%$ \\
\hline
\end{tabular}

Legenda: 0 - não existem planos de implantação; 1 - planejada para o futuro; 2 - em processo de implantação; 3 - já está implantada; 4 - implantada e apresentando resultados relevantes, e $n / r$ - não responderam.

Fonte: Elaborada pelos autores (2017).

Pela Tabela 1 apresentada, verifica-se pela análise das médias que, de forma geral, as práticas de GC de base tecnológica e funcional abordadas possuem o nível efetivo de implantação alto. Entre os gestores da empresa pesquisada, $76 \%$ avaliaram que estas práticas estão implementadas entre os níveis 3 (a prática já está implantada) e 4 (a prática já está implantada e apresenta resultados importantes e relevantes para a organização). Os demais gestores consideraram que as práticas encontram-se nos seguintes níveis de implantação: $17 \%$ dos gestores responderam que as práticas se limitam ao nível 2, ou seja, ainda estão em processo de implementação; $1 \%$ dos gestores responderam que existem determinadas ações planejadas para implementar futuramente (nível 1); 4\% dos gestores não se posicionaram quanto ao nível das práticas; e os outros $1 \%$ dos gestores tem a percepção que não há planos para implementação, ou seja, encontra-se em nível 0 .

$\mathrm{Na}$ análise individual de cada prática, todas apresentaram nível efetivo de implantação alto (soma dos níveis 3 e 4) pela percepção dos gestores: portais/intranets/extranets (93\%); Sistema de Workflow (71\%); Gestão Eletrônica de Documentos (64\%); CRM (86\%); e acerca da prática ERP (os gestores 64\%), identificaramno com o Software RIS, cuja funcionalidade é a mesma. Contudo, consideram o RIS mais completo e integrado do que o ERP, conforme análise documental.

No Gráfico 1 apresentam-se os resultados quanto ao nível efetivo de implantação das práticas de GC de base tecnológica e funcional. 
Gráfico 1 - Nível efetivo de implementação das práticas de base tecnológica e funcional na empresa Clínica de Diagnóstico por Imagem

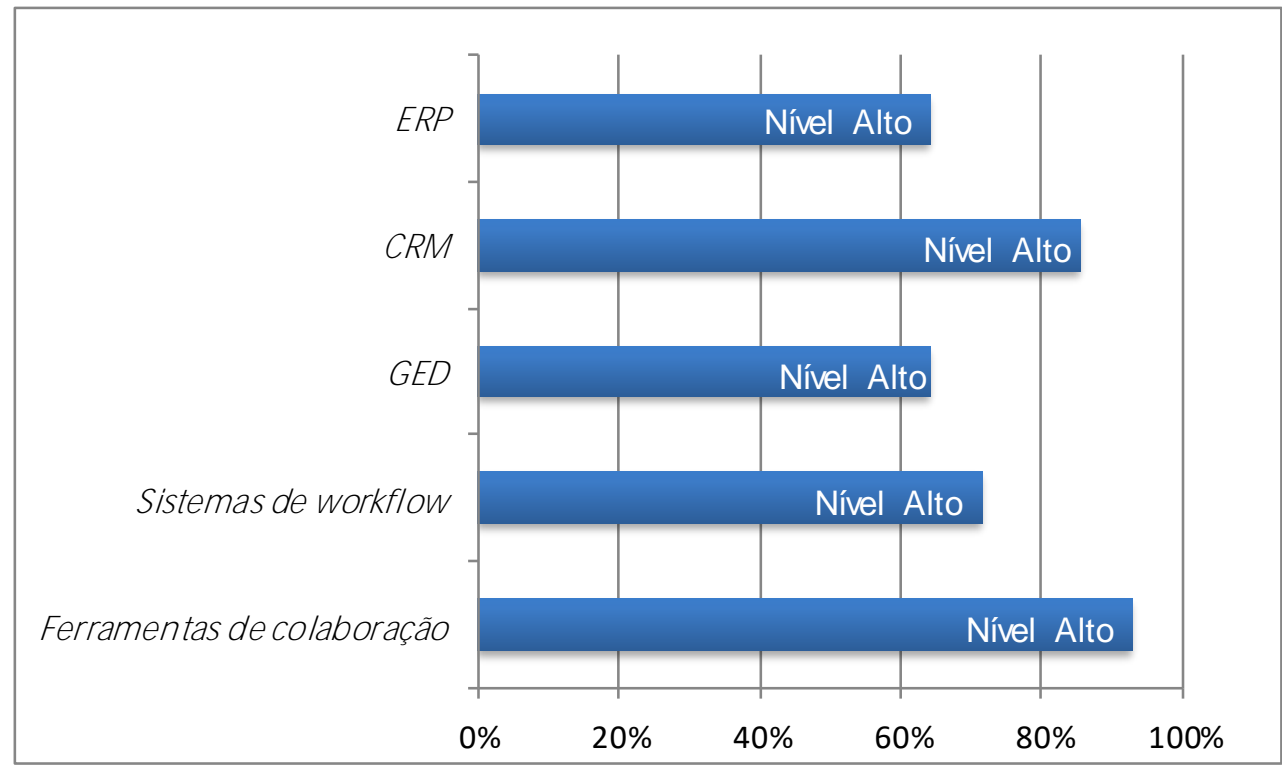

Legenda: 0 - não existem planos de implantação; 1 - planejada para o futuro; 2 - em processo de implantação; 3 - já está implantada; 4 - implantada e apresentando resultados relevantes, e n/r - não responderam.

Fonte: Elaborada pelos autores (2017).

Quanto ao nível de amplitude do alcance, registram-se na Tabela 2 os resultados das práticas de GC em relação ao seu uso na empresa Clínica de Diagnóstico por Imagem.

Tabela 2 - Nível de alcance das práticas de GC de base tecnológica e funcional

\begin{tabular}{|l|c|c|c|c|c|c|}
\hline \multicolumn{1}{|c|}{ PRÁTICAS } & \multicolumn{3}{c|}{ Alcance } & \multicolumn{3}{c|}{} \\
\cline { 2 - 6 } \multicolumn{1}{|c|}{} & $\mathbf{1}$ & $\mathbf{2}$ & $\mathbf{3}$ & $\mathbf{4}$ & $\mathbf{N} / \mathbf{R}$ \\
\hline Ferramentas de colaboração & $0 \%$ & $7 \%$ & $64 \%$ & $29 \%$ & $0 \%$ \\
\hline Sistemas de workflow & $7 \%$ & $14 \%$ & $21 \%$ & $50 \%$ & $7 \%$ \\
\hline Gestão Eletrônica de Documentos (GED) & $0 \%$ & $21 \%$ & $50 \%$ & $29 \%$ & $0 \%$ \\
\hline Costumer Relationship Management (CRM) & $0 \%$ & $7 \%$ & $43 \%$ & $43 \%$ & $7 \%$ \\
\hline Enterprise Resource Planning (ERP) & $0 \%$ & $36 \%$ & $36 \%$ & $21 \%$ & $7 \%$ \\
\hline
\end{tabular}

Legenda: 1 - poucas iniciativas isoladas; 2 - restrito a alguns departamentos; 3 - muitos departamentos usam a prática; 4 - amplamente disseminada na organização, e N/R - não responderam.

Fonte: Elaborada pelos autores (2017).

No que se refere a Tabela 2, aponta-se a ocorrência de maneira predominante de altos níveis de percepção pelos gestores quanto ao nível de amplitude do alcance (soma dos níveis 3 e 4) para maioria das práticas de GC de base tecnológica e funcional abordadas no estudo. De forma em geral, a média apresentada para a empresa Clínica de Diagnóstico por Imagem foi de $77 \%$, sendo os demais $22 \%$ estão distribuídos da seguinte forma: 17\% para nível 2 (alguns departamentos usam a prática); 1\% para nível 1 (poucas iniciativas isoladas dentro da organização); e 4\% corresponde os gestores que não opinaram.

As práticas apresentadas com nível de amplitude do alcance alto (soma dos níveis 3 e 4) conforme a percepção pelos gestores foram as seguintes: portais/intranets/extranets (93\%), Sistemas de Workflow (71\%), GED (79\%) e CRM (86\%). A prática ERP foi a única que apresentou nível de amplitude do alcance mediano (57\%), o qual os gestores identificaram como Software RIS.

Por meio do Gráfico 2 verifica-se a percepção dos gestores quanto ao nível de amplitude do alcance das práticas de GC de base tecnológica e funcional. 
Gráfico 2 - Nível de amplitude do alcance das práticas de base tecnológica e funcional na empresa Clínica de Diagnóstico por Imagem

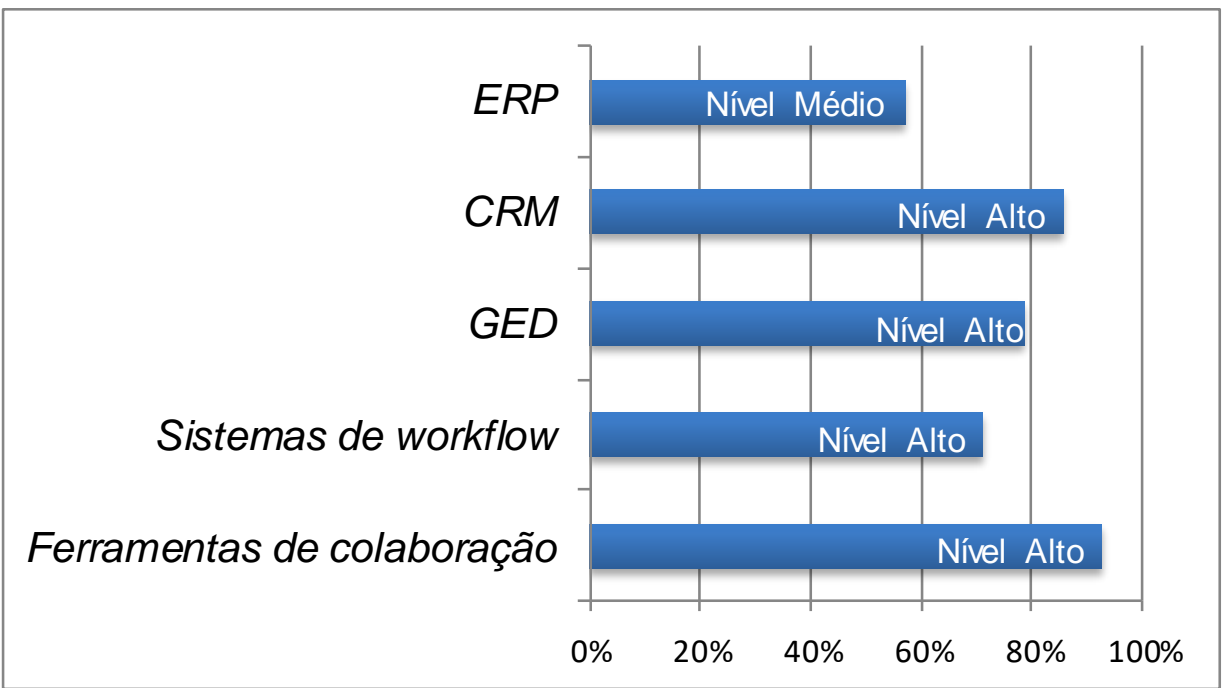

Legenda: 1 - poucas iniciativas isoladas; 2 - restrito a alguns departamentos; 3 - muitos departamentos usam a prática; 4 - amplamente disseminada na organização, e $n / r$ - não responderam. Fonte: Elaborada pelos autores (2017).

Para melhor compreensão dos resultados, no Gráfico 3 apresenta-se uma análise comparativa dos níveis de amplitude do alcance em relação aos níveis efetivos de implementação das práticas de GC de base tecnológica e funcional. Posteriormente, abordaram-se as possíveis implicações e benefícios dessas práticas na empresa pesquisada.

Gráfico 3 - Percepção Integrada acerca do nível de implantação e alcance das práticas de GC de base tecnológica e funcional

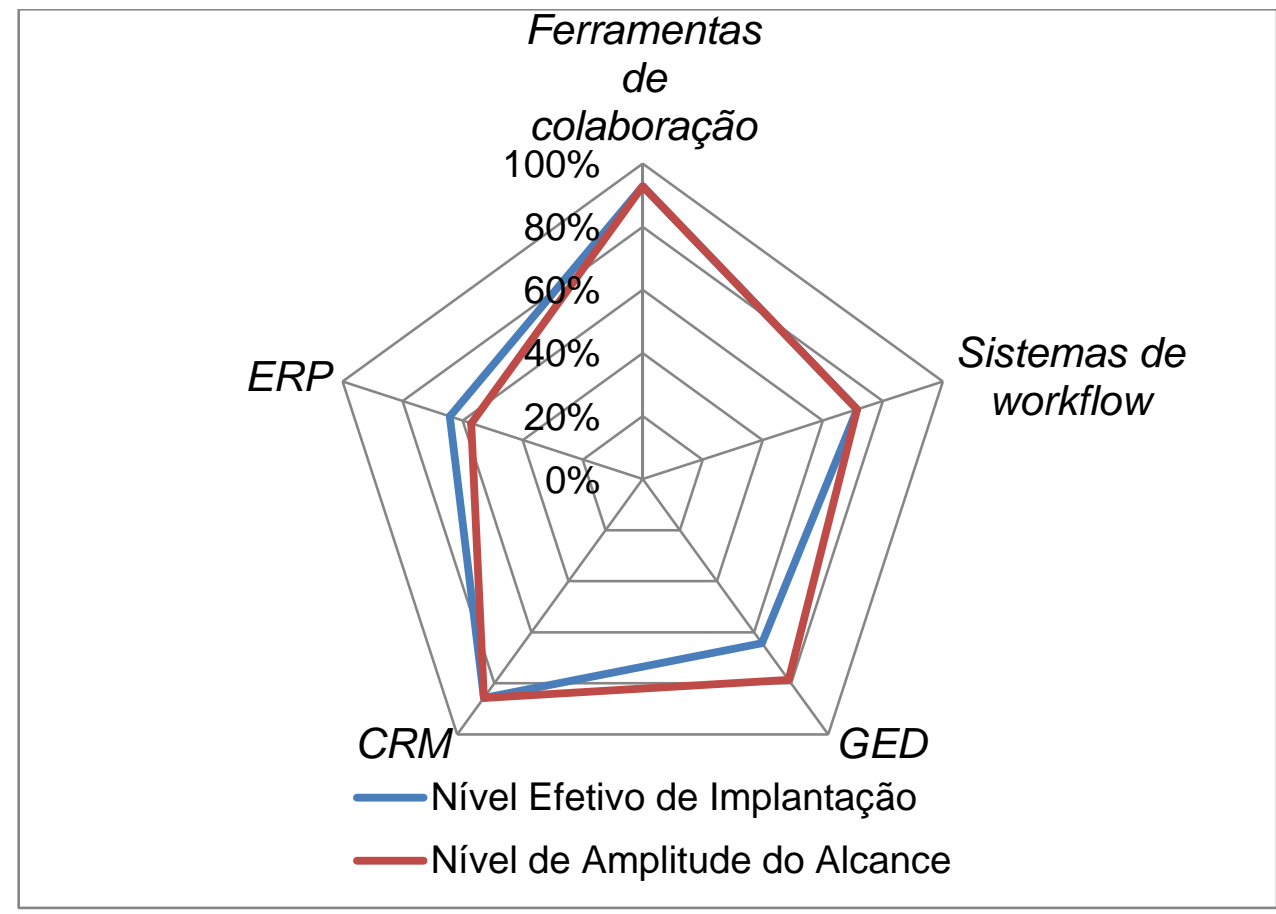

Fonte: Elaborada pelos autores (2017). 
De maneira em geral, pelo Gráfico 3, verifica-se que os níveis de amplitude do alcance das práticas de GC de base tecnológica e funcional acompanham os níveis efetivos de implementação para maioria das práticas. A predominância do nível de amplitude do alcance das práticas de GC abordadas nesse estudo varia entre o nível médio e alto. Portanto, observa-se que as práticas de GC estão sendo utilizadas na empresa, aproxima-se do total de suas potencialidades.

Ainda conforme Gráfico 3, examina-se que a percepção (em quantitativo) dos gestores sobre o nível de amplitude do alcance é o mesmo para o nível efetivo de implantação para as práticas Portais/Intranets/Extranets, Sistemas de Workflow e CRM. Logo, tem-se o seguinte resultado para essas práticas: $93 \%, 71 \%$ e $86 \%$ respectivamente, resultados que representam níveis altos e, diante disso, observase que estas práticas estão sendo empregadas próximo do total de suas potencialidades.

Entende-se que os benefícios que podem ser gerados a partir da existência da prática de portais/intranets/extranets são: melhor captura e difusão do conhecimento; além do espaço para compartilhar as experiências entre os colaboradores; redução de processos e tempo uma vez que proporciona aplicações relevantes e acesso a todas as informações para essa empresa; e também exerce a função de plataforma para outras práticas, tais como: redes do Conhecimento, comunidades de prática e melhores práticas (QUINN et al., 2014).

Já como benefícios que podem ser gerados pela prática Sistemas de Workflow à empresa em questão, podem-se citar: maior qualidade no controle interno; maior agilidade e simplificação nos trabalhos diários pelos os funcionários; eficiência no controle da qualidade da informação, contribuindo principalmente para a parte operacional, por exemplo, controle entre pedidos efetuados pelos médicos e entrega de exames aos pacientes.

Em relação à prática CRM, têm-se os seguintes benefícios: registro de outras informações que os gestores conseguem realizar para acompanhar a satisfação do paciente como a qualidade dos serviços oferecidos de exames de imagem; registro de informações de compra de materiais/manutenção de equipamentos e prazos de entrega; registro da satisfação dos médicos quanto aos laudos realizados pelos profissionais da área técnica pela empresa.

Também se observa no Gráfico 3 que a GED está sendo utilizada próximo do total de sua potencialidade, o que pode proporcionar os seguintes benefícios: adquirir aplicativos informatizados que contribuem na qualidade do controle interno das informações entre os departamentos; agilidade em localizar documentos nesses aplicativos; redução impressos e consequentemente na redução de gastos com materiais de escritório.

No caso da prática de GC de ERP, o nível de implementação foi alto e o nível de amplitude do alcance foi mediano, aponta-se que o RIS está sendo utilizado abaixo de sua potencialidade na empresa, sendo necessário propor medidas para sua ampliação. 0 pouco uso da dessa prática pode acarretar prejuízos para à empresa, tais como: tecnologia da Informação pouco eficaz; dificuldade em gerar e disseminar o conhecimento entre os colaboradores, ocasionando danos na informação e excesso de retrabalhos e gastos desnecessários com outros sistemas de informação.

\section{CONCLUSÃO}

O objetivo geral proposto para esse estudo foi diagnosticar o nível de implantação e de alcance de práticas de GC de base tecnológica e funcional em uma empresa do ramo de saúde que realiza exames de diagnóstico por imagem no município de Maringá (PR).

0 resultado do coeficiente Alfa de Cronbach encontrado foi excelente tanto para o nível de implementação quanto para o nível de alcance das práticas de GC contidas no questionário, permitindo deduzir que há confiabilidade nos dados obtidos pela aplicação do questionário, descartando a necessidade de aplicar o método de purificação da escala.

O resultado do diagnóstico revelou que as práticas de GC de base tecnológica e funcional apresentaram um nível efetivo de implantação alto, pois, $76 \%$ dos gestores, na média, consideraram que as práticas dessa categoria se encontram entre o nível 3 (a prática já está implantada) e o nível 4 (a prática já está 
implantada e apresenta resultados importantes e relevantes para a organização). Todas as práticas obtiveram nível efetivo de implantação alto.

O nível de amplitude do Alcance para essa categoria também é considerado alto, tendo-se em vista que, na média, 77\% dos gestores responderam que as práticas permeiam entre 0 nível 3 (muitos departamentos usam a prática) e nível 4 (amplamente disseminada na organização) na empresa pesquisada. Das práticas desta catego ria, apenas ERP foi classificada com nível de amplitude do Alcance médio, enquanto todas as demais práticas foram avaliadas com nível de amplitude do Alcance alto.

Os gestores identificaram que o software RIS substitui a prática ERP, pois o mesmo é muito mais completo, além de apresentar as mesmas funcionalidades. No entanto, essa prática está sendo utilizada abaixo de sua potencialidade, sugerindo-se que a empresa disponibilize treinamentos e cursos para que os seus colaboradores e gestores possam aprender as funcionalidades e importância desse recurso. Isso poderia resultar na utilização da prática de acordo com todas as suas potencialidades e, consequentemente, melhorar a tomada de decisões na empresa. A elaboração de manuais com linguagem menos técnica também seria outra importante ação que facilitaria a utilização desse software dentro de suas potencialidades. Por fim, sugere-se também a contratação de um Coaching que possa, no dia a dia, incentivar e contribuir para que o RIS seja plenamente utilizado. Verificou-se também que por meio da análise documental que a empresa possui redes so ciais, tais como Facebook, Instagram, Linkedin e o próprio site, local em que disponibiliza informações, principalmente aos pacientes, tais como: serviços oferecidos, resultados de exames e agendamento on line.

Faz-se importante destacar que para a elaboração desta pesquisa foi necessário lidar com algumas limitações e barreiras que surgiram ao longo do processo. Umas das principais dificuldades enfrentadas foi com a não permissão para o acesso a dados estratégicos da empresa pesquisada, bem como acesso a informações específicas de alguns setores, tais como o departamento financeiro e o setor onde realizam os exames de imagem, além do departamento contábil, o qual não se localiza em nenhuma das unidades, pois a contabilidade é realizada por profissional externo.

Como desdobramento futuros, pretende-se elaborar um relatório para entregar para a empresa, a fim de contribuir na consolidação da GC. Pretende-se também realizar uma análise de todas as práticas, de acordo com o quadro 2, para uma análise e diagnóstico mais realístico do nível de maturidade de GC da empresa. Também como desdobramentos futuros, há a intenção de dar continuidade a essa pesquisa, aplicando o mesmo instrumento de diagnóstico em outras organizações desse ramo, uma vez que a GC é um tema ainda pouco explorado nestas organizações no município de Maringá. A aplicação do diagnóstico em outras organizações do ramo de saúde também permitirá a elaboração de análises comparativas, com os dados obtidos nesta pesquisa.

\section{REFERÊNCIAS}

AVEDON, D. M. GED de A a Z: tudo sobre gerenciamento eletrônico de documentos. Tradução Roberta da Silva Aquino. São Paulo: CENADEM, 2002.

BATISTA, F. F. 0 Desafio da GC nas áreas de administração e planejamento das instituições federais de ensino superior. Brasília: IPEA, Maio, 2006. Disponível em:

http://www.ipea.gov.br/portal/images/stories/PDFs/TDs/td_1181.pdf. Acesso em: 1 jul. 2016.

CARVALHO, F. C. A. Gestão do Conhecimento. 5. ed. São Paulo: Pearson, 2012.

DALKIR, K. Knowledge management in theory and practive. Burlington: Elsevier, 2005. K. Knowledge management in theory and practive. Burlington: Elsevier, 2011

DAVENPORT, T. H; PRUSAK, L. Conhecimento empresarial: como as organizações gerenciam seu capital intelectual. Rio de Janeiro: Campus, 1999. 
DUARTE, E. N. et al. Vantagens do uso de tecnologias para criação, armazenamento e disseminação do conhecimento em bibliotecas universitárias. Transinformação, Campinas, v. 18, n. 2, p. 131-141, maio/ago. 2006. Disponível em: http://periodicos.puc-campinas.edu.br/seer/index.php/transinfo/article/view/675/655. Acesso em: 1 jul. 2016.

DRUCKER, P. F. Prática da Administração de Empresas. São Paulo: Pioneira Thomson, 1981.

HORA, H. R. M.; MONTEIRO, G. T. R.; ARICA, J. Confiabilidade em questionário para qualidade: um estudo com o Coeficiente Alfa de Cronbach. Produto \& Produção, v. 11, n. 2, p. 85 - 103, jun. 2010.

INFOPÉDIA. Dicionários Porto Editora. 2016. Disponível em: http://www.infopedia.pt/dicionarios/linguaportuguesa/pr\%C3\%Altica. Acesso em: 3 jul. 2017.

LOESCH, C.; HOELTGBAUM , M. Métodos estatísticos multivariados. São Paulo: Saraiva, 2012.

MATTHIENSEN, A. Uso do coeficiente Alfa de Cronbach em avaliações por questionários. Boa Vista: Embrapa, 2011.

MENDES, J. V.; ESCRIVÃO FILHO, E. Sistemas integrados de gestão ERP em pequenas empresas: um confronto entre o referencial teórico e a prática empresarial. Gestão \& Produção, São Carlos, v. 9, n. 3, p. 277-296, 2002. Disponível em: http://www.scielo.br/scielo.php?script=sci_arttext\&pid=S0104-

530X2002000300006\&lng=pt\&nrm=iso\&tIng=pt. Acesso em: 25 out. 2016.

QUINN, E. et al. How can knowledge exchange portals assist in Knowledge management for evidenceinformed decision making in public health? BioMed Central, Londres, v. 14, n. 443, p. 2-8, 2014. Disponível em: http://bmcpublichealth.biomedcentral.com/articles/10.1186/1471-2458-14-443. Acesso em: 23 nov. 2016

RODRIGUEZ, M. V. R. Gestão do conhecimento e inovação nas empresas. Rio de Janeiro: Qualitymark, 2011.

ROESE, A.; LADEIRA JÚNIOR, W. Gerenciamento eletrônico de documentos: alternativas tecnológicas para GC. In: ENCONTRO DE ADMINISTRAÇÃO DA INFORMAÇÃO (EnADI), 2., Recife, 2009. Atas do II Encontro da EnADI. Recife, Pernambuco: ANPAD, 2019. p. 1-16.

ROSENBERG, G.; OHAYON, P.; BATISTA, F. F. Gestão do Conhecimento em organizações públicas de saúde no Brasil: diagnóstico de práticas. Revista do Serviço Público, Braślia, v. 59, n. 1, p. 43-60, jan./mar. 2008.

SOUZA, M. A. B. Gestão do conhecimento: uma contribuição ao seu entendimento. Revista de Administração e Negócios da Amazônia, Porto Velho, v. 6, n. 3, p. 38-47; set./dez. 2014.

STEIL, A. V. Estado da arte das definições de gestão do conhecimento e seus subsistemas. Florianópolis: Instituto Stela, 2007.

TARAPANOFF, K. Informação, conhecimento e inteligência em corporações: relações e complementaridade. In:TARAPANOFF, Kira. (org). Inteligência, Informação e Conhecimento. Braślia: IBICT; UNESCO, 2006. p. 1935.

URIARTE J r., Filemon A. Introduction to knowledge management: a brief introduction to the basic elements of knowledge management for non-practitioners interested in understandiong the subject. Indonésia: Asean Foundation, 2008.

YOUNG, R. Knowledge management: tools and techniques manual. United Kingdom: Asian Productivity Organization (APO), 2010. 